\title{
BMJ Global Health Reduction in healthcare services during the COVID-19 pandemic in China
}

\author{
Yi-Na Zhang, ${ }^{1}$ Yun Chen (10,${ }^{2}$ Ying Wang ${ }^{2}$ Fan Li, ${ }^{3}$ Michelle Pender, ${ }^{4}$ Na Wang, ${ }^{2}$ \\ Fei Yan, ${ }^{2}$ Xiao-Hua Ying, ${ }^{2}$ Sheng-Lan Tang, ${ }^{4}$ Chao-Wei Fu ${ }^{2}$
}

\begin{abstract}
To cite: Zhang Y-N, Chen Y, Wang $Y$, et al. Reduction in healthcare services during the COVID-19 pandemic in China. BMJ Global Health 2020;5:e003421. doi:10.1136/ bmjgh-2020-003421
\end{abstract}

\section{Handling editor Sanni Yaya}

- Additional material is published online only. To view, please visit the journal online (http://dx.doi.org/10.1136/ bmjgh-2020-003421).

Y-NZ and YC are joint first authors.

Received 11 July 2020 Revised 2 October 2020 Accepted 6 October 2020

Check for updates

(c) Author(s) (or their employer(s)) 2020. Re-use permitted under CC BY-NC. No commercial re-use. See rights and permissions. Published by BMJ.

${ }^{1}$ Department of Socail Management and Socail Policy, School of Social Development and Public Policy, Fudan University, Shanghai, China

${ }^{2}$ Key Laboratory of Public Health Safety, NHC Key Laboratory of Health Technology Assessment, School of Public Health, Fudan University, Shanghai, China ${ }^{3}$ Department of Statistical Science, Duke University, Durham, North Carolina, USA ${ }^{4}$ Duke Global Health Institute, Duke University, Durham, North Carolina, USA

\section{Correspondence to}

Dr Chao-Wei Fu;

fcw@fudan.edu.cn and

Dr Xiao-Hua Ying;

xhying@fudan.edu.cn

\section{ABSTRACT}

Introduction The COVID-19 pandemic caused a healthcare crisis in China and continues to wreak havoc across the world. This paper evaluated COVID-19's impact on national and regional healthcare service utilisation and expenditure in China.

Methods Using a big data approach, we collected data from 300 million bank card transactions to measure individual healthcare expenditure and utilisation in mainland China. Since the outbreak coincided with the 2020 Chinese Spring Festival holiday, a difference-indifference (DID) method was employed to compare changes in healthcare utilisation before, during and after the Spring Festival in 2020 and 2019. We also tracked healthcare utilisation before, during and after the outbreak. Results Healthcare utilisation declined overall, especially during the post-festival period in 2020. Total healthcare expenditure and utilisation declined by $37.8 \%$ and $40.8 \%$, respectively, while per capita expenditure increased by $3.3 \%$. In a subgroup analysis, we found that the outbreak had a greater impact on healthcare utilisation in cities at higher risk of COVID-19, with stricter lockdown measures and those located in the western region. The DID results suggest that, compared with low-risk cities, the pandemic induced a $14.8 \%, 26.4 \%$ and $27.5 \%$ reduction in total healthcare expenditure in medium-risk and high-risk cities, and in cities located in Hubei province during the postfestival period in 2020 relative to 2019 , an $8.6 \%, 15.9 \%$ and $24.4 \%$ reduction in utilisation services; and a $7.3 \%$ and $18.4 \%$ reduction in per capita expenditure in mediumrisk and high-risk cities, respectively. By the last week of April 2020, as the outbreak came under control, healthcare utilisation gradually recovered, but only to $79.9 \%-89.3 \%$ of its pre-outbreak levels.

Conclusion The COVID-19 pandemic had a significantly negative effect on healthcare utilisation in China, evident by a dramatic decline in healthcare expenditure. While the utilisation level has gradually increased post-outbreak, it has yet to return to normal levels.

\section{INTRODUCTION}

COVID-19, the infectious disease caused by the novel coronavirus SARS-COV-2 was first identified in Wuhan, China, in December $2019^{1}$ and has rapidly become the worst global health pandemic in a century. As of 2 September 2020, there are 25.6million

\section{Key questions}

What is already known?

- There have not been any previous research studies which have quantified and tracked the impact of COVID-19 on the utilisation of healthcare services in China.

What are the new findings?

- Our findings show that the COVID-19 pandemic had a significant negative effect on healthcare service utilisation in China, regardless of the level of risk in cities, emphasising the national and global impact of pandemics. While the healthcare service utilisation level has gradually increased during the post-outbreak period, it has yet to return to the preoutbreak level, nor does it appear to be on track to do so.

What do the new findings imply?

- Our findings suggest that countries need to find ways to minimise the disruption of healthcare services for non-pandemic-related health concerns as much as possible, and find ways to quickly return to pre-pandemic levels.

confirmed cases including 852758 deaths in over 200 countries and territories. ${ }^{2}$ In China, COVID-19 caused 84081 confirmed cases and 4638 deaths. ${ }^{3}$ In response to the outbreak, China enacted strict 'lockdown' policies to prevent or slowdown person-to-person transmission. Mass gatherings and public events were banned; schools, public buildings and businesses were closed; and all travel was restricted. ${ }^{4}$ As a result of these restrictions, many patients were unable to access healthcare services. Furthermore, many outpatient and inpatient services were suspended as healthcare workers and other health resources were reallocated to COVID-19. In addition, patients avoided seeking healthcare for fear of contracting the virus in healthcare settings. This disruption in services left many patients without access to necessary healthcare. ${ }^{56}$ There have been reports of patients having to delay necessary healthcare such 
as surgery, dialysis and cancer treatment. ${ }^{5-9}$ A study in Germany found that physical rehabilitation and postoperative follows-up were severely affected by COVID-19. ${ }^{8}$ Yet another study in Dubai reported that the pandemic has significantly affected children with cochlear implants. ${ }^{9}$ However, these studies were based on simple observation designs with limited ability to capture causal relationship.

Previous public health emergences have resulted in similar reductions in healthcare service utilisation for similar reasons. For example, studies have shown that facility-based healthcare, maternal and child healthcare, vaccination services and HIV/AIDS treatment all declined during the 2014-2015 Ebola epidemic in West Africa $^{10-14}$ and were sometimes slow to return to normal levels. $^{15}$

We employed a big data approach to assess the impact of the COVID-19 outbreak on healthcare service utilisation at both national and regional levels in China. Our in-depth examination of the negative impact caused by COVID-19 will help to improve our understanding of the scope of such public health emergencies and underscore the importance of strengthening health systems. As countries across the world continue to battle the pandemic and are forced to enact similar mitigating measures and reallocate resources to patients with COVID-19, we could expect to see similar patterns in healthcare service utilisation in other countries, as well as lingering patterns in China. ${ }^{16}$

\section{METHODS \\ Healthcare expenditure and healthcare service utilisation data}

Healthcare expenditure was measured using financial transactions from China UnionPay (CUP) bank cards. CUP is a financial services corporation that provides bank cards in mainland China. In 2019, over 8 billion CUP cards has been issued through 400 financial institutions and accepted in more than 170 countries, and CUP is the most popular bank card in China and is accepted by most merchants and ATMs. ${ }^{17}$ CUP transaction database keeps track of every transaction for the CUP card users, and data are categorised by type of industry, such as retail, medical and financial. CUP cards are the most common form of payment for non-reimbursable healthcare services in China, and all healthcare-related payments are processed through the CUP medical category. The CUP medical database keeps track of daily transactions for healthcare services including date, amount, location and name of the healthcare facility (including hospitals and community healthcare centres). The dataset we used included healthcare expenditure information for CUP card users in 365 mainland cities and 31 provinces in China during 2019 and 2020.

Given that the COVID-19 pandemic occurred during the Chinese Spring Festival holiday, we selected the time before, during and after the holiday as our study period. This timeline included 9 weeks in 2019 and 2020 and covered the pre-festival period (4 weeks before the Spring Festival holiday), the festival itself (1 week) and the post-festival period (4weeks after the Spring Festival holiday). The study period in 2019 fell between 8 January and 12 March 2019, and between 28 December 2019 and 28 February 2020. To further capture patterns in healthcare service utilisation, we also collected data between 1 November 2019 and 30 April 2020 (the time before, during and after the outbreak). This included the preoutbreak (weeks 1 to 12: 1 November 2019 to 22 January 2020), outbreak (weeks 13 to 16: 23 January 2020 to 20 February 2020) and post-outbreak (weeks 17 to 26: 21 February 2020 to 30 April 2020) periods. Data were collected on total healthcare expenditure, utilisation frequency and per capita expenditure for each city. Total healthcare expenditure was defined as the average daily total expenditure on healthcare services within a week; utilisation frequency was defined as the average daily number of times people used healthcare services within a week; and per capita expenditure was defined as total healthcare expenditure divided by the total number of times people used healthcare services within a week, in other words, the average amount of money spent on a single healthcare service. Data included 300 million transactions amounting to $¥ 350$ billion (US $\$ 49.4$ billion).

In recent years, new financial transaction mechanisms such as WeChat Pay and Ali Pay have become more common in China; as a result, the market share of CUP cards decreased between 2019 and 2020; however, the degree of decrease specific to the medical industry is unknown. As a result, we were unable to use the CUP data to directly compare utilisation between 2019 and 2020 . Instead, we used the relative values of total healthcare expenditure, utilisation frequency or per capita expenditure as the outcomes, defined as the ratio between the value of each outcome in a given week and the first week of the same year. The change in those relative values may capture the same change as the true values in healthcare utilisation between 2019 and 2020. Initially 365 cities were included in data collection, but two cities were excluded due to the lack of available data. As a result, a total of 363 cities were included in the final analysis.

\section{COVID-19 outbreak data}

Data on the number of COVID-19 cases, deaths and recoveries were collected from the local Centers for Disease Control and Prevention in all 365 cities, which recorded daily figures for each city between 24 January and 26 February 2020. Cities were classified into four groups (online supplemental figure S1) based on their cumulative COVID-19 confirmed cases. The groups are the following: low-risk cities (case number $<10$ ); mediumrisk cities $(10 \leq$ case number $<100)$; high-risk cities (case number $\geq 100$ ) and cities located in Hubei province, the epicentre of the COVID-19 outbreak in China. A second classification divided the cities into lockdown and non-lockdown categories. Lockdown status was classified using the following factors: (1) prohibition of 
unnecessary commercial activities, (2) prohibition of any type of public or private gathering and (3) restrictions on private and public transportation. Lockdown factors were measured on a daily basis. A city was classified as being in lockdown if the aforementioned factors were implemented for over half of the week (online supplemental table S1). ${ }^{18}$

\section{Ethics statement}

This study did not include any human subjects or animals.

\section{Patient and public involvement}

There was no patient involvement.

\section{Statistical analysis}

To account for inflation while calculating total healthcare expenditure, we recalculated the absolute value of healthcare expenditure between 8 January and 12 March 2019 by multiplying the average consumer price index from January to February 2020 (5.3\%). We used the difference-in-differences (DID) method to quantify the impact of COVID-19 on healthcare service utilisation in different risk groups. ${ }^{19}$ Specifically, we postulated the following DID model:

$$
Y_{i, t}=\beta_{0}+\beta_{1} \text { Treat }_{i}^{*} \text { Week }_{t}^{1}+\beta_{2} \text { Treat }_{i}^{*} \text { Week }_{t}^{2}+\beta_{3} \text { Treat }_{i}^{*} \text { Week }_{t}^{3}+d_{i}+d_{t}+\varepsilon_{i, t}
$$

where $i(i=1, \ldots, 363)$ denotes the city and $t(t=-9, \ldots,-1$, $0,1, \ldots, 8)$ denotes the week. Week ${ }_{t}^{1}$, Week ${ }_{t}^{2}$ and $W e e k_{t}^{3}$ are dummy variables for week $t$ which equals the pre-festival period in $2020(t=1, \ldots, 3)$, during the festival period in $2020(t=4)$ and the post-festival period in $2020(t=5, \ldots$, 8 ) and equals zero otherwise. The omitted benchmark period includes weeks in $2019(t<0)$, including the prefestival period in $2019(\leq-6)$, during the festival in 2019 $(t=-5)$ and the post-festival period in $2019(t=-4, \ldots,-1)$. The dependent variable, $Y_{i, t}$ is healthcare service utilisation in city $i$ during week $t$. $d_{i}$ and $d_{t}$ are vectors of the city and week dummy variables that account for city and week fixed effects. The city-specific fixed effects $d_{i}$ control for time-invariant, unobserved city-specific heterogeneities. The week-specific fixed effects $d_{t}$ control for unobserved time-specific factors, including travel related to the Spring Festival (a time with high rates of travel in China). Definition of the treatment variable Treat $_{i}$ varies by specific DID design. We conducted three separate DID analyses. All used low-risk cities as the control group (ie, Treat=0), and the treatment groups were (ie, Treat $=1$ ) (Panel A) medium-risk cities; (Panel B) high-risk cities and (Panel C) cities located in Hubei. The coefficients of the city-by-week interaction, $\beta_{1}, \beta_{2}$ and $\beta_{3}$ are the target parameters of the DID model which estimates the differences in healthcare service utilisation between treatment and control groups before, during and after the Spring Festival in 2020 relative to 2019. A positive and significant $\beta_{1}, \beta_{2}$ and $\beta_{3}$ suggest that COVID-19 exerted a positive effect on healthcare service utilisation, while a negative and significant $\beta_{1}, \beta_{2}$ and $\beta_{3}$ indicate that COVID-19 reduced healthcare service utilisation.
The parallel-trend assumption and dynamics of the relationship between COVID-19 and healthcare service utilisation underlying the DID estimators were tested according to Jacobson $e t a l .{ }^{20}$ Specifically, we added a set of dummy variables to the standard model (1) to trace the week-by-week effects of the pandemic on healthcare service utilisation:

$Y_{i, t}=\beta_{0}+\beta_{1}$ Treat $_{i}^{*}$ Week $_{t}^{1}+\beta_{2}$ Treat $_{i}^{*}$ Week $_{t}^{2}+\ldots+\beta_{17}$ Treat $_{i}^{*}$ Week $_{t}^{17}+d_{i}+d_{t}+\varepsilon_{i, t}$

where the added variables Week $_{t}^{j}(j=1, \ldots, 17)$ are the dummy variables of week $j$ that equals 1 when week $t=j$ and equals 0 otherwise, and the omitted benchmark week is the first week in 2019 (ie, $t=-9$ ). The coefficient of the cityby-week interaction, $\beta$, estimates the dynamic effect of COVID-19 on healthcare service utilisation between the treatment group and the control group in week $t$ relative to the first week of 2019. If the parallel trend assumption holds, $\beta_{t}$ would be close to 0 when week $t$ is in 2019. All analyses were performed using Stata 16.0 for Windows statistical software (Stata, College Station, Texas, USA). The maps were plotted using $\mathrm{R}$ 4.0.0. ${ }^{21}$

\section{RESULTS \\ Percentage change in healthcare utilisation in 2020 compared with 2019}

In online supplemental table S2, we show the absolute value for total healthcare expenditure, utilisation frequency and per capita expenditure in the pre-festival, Spring Festival and post-festival periods between 2019 and 2020. Compared with 2019, the average healthcare expenditure and utilisation frequency in 363 cities decreased in 2020. The percentage change was highest during the post-festival period, during which total healthcare expenditure decreased by $55.1 \%$ (95\% CI: $-61.3 \%$ to $-48.8 \%$ ) and utilisation frequency decreased by $67.2 \%$ (95\% CI: $-68.7 \%$ to $-65.7 \%$ ). Compared with 2019 , the average per capita expenditure was lower for all 363 cities in 2020 except during the post-festival period.

In table 1, we show the relative value for healthcare service utilisation before, during and after the Spring Festival in 2019 and 2020. Compared with 2019, the average healthcare service utilisation in 363 cities increased during the pre-festival period in 2020. The percentage change in total expenditure, utilisation frequency and per capita expenditure was $5.4 \%$ (95\% CI: $-1.0 \%$ to $11.7 \%$ ), $2.5 \%$ (95\% CI: $1.3 \%$ to $3.6 \%$ ) and $1.3 \%$ ( $95 \%$ CI: $-1.7 \%$ to $4.4 \%$ ), respectively. During and after the festival, total expenditure and utilisation frequency declined, the greatest percentage change occurred during the post-festival period when total expenditure and utilisation frequency decreased by $37.8 \% \quad(95 \%$ CI: $-42.9 \%$ to $-32.7 \%$ ) and $40.8 \%(95 \%$ CI: $-42.0 \%$ to $-39.5 \%)$, respectively. During the festival week in 2020, per capita expenditure decreased by $5.5 \%$ (95\% CI: $-10.0 \%$ to $-1.0 \%$ ) compared with the same period in 
2019 and increased by $3.3 \%$ (95\% CI: $-1.0 \%$ to $7.6 \%$ ) after the festival.

Percentage change in healthcare utilisation between different subgroups

Table 1 also shows the percentage change in healthcare service utilisation in the different subgroups. Compared with the same period in 2019, total healthcare expenditure and utilisation frequency decreased in medium-risk and high-risk cities and in cities located in Hubei province during and after the Spring Festival in 2020. The percentage change in total expenditure and utilisation frequency were greatest in high-risk cities $(60.6 \%, 95 \% \mathrm{CI}$ : $-63.0 \%$ to $-58.1 \%$ ) and cities located in Hubei province $(58.0 \%, 95 \%$ CI: $-63.7 \%$ to $-52.2 \%)$, respectively. In lowrisk cities, the percentage change in total expenditure and utilisation frequency increased by $18.2 \%$ (95\% CI: $-2.3 \%$ to $34.0 \%$ ) and $7.4 \%$ (95\% CI: $4.8 \%$ to $10.0 \%$ ) before the Spring Festival and decreased by $26.1 \%$ (95\% CI: $-30.9 \%$ to $-21.2 \%$ ) and $32.8 \%$ (95\% CI: $-35.4 \%$ to $-30.2 \%$ ) after the Spring Festival in 2020, respectively. Per capita expenditure decreased in medium-risk and high-risk cities and increased in low-risk cities and cities located in Hubei province after the festival in 2020 compared with the same period in 2019. Compared with 2019, the total healthcare expenditure and utilisation frequency decreased and the greatest percentage change occurred in higher lockdown cities in 2020. In lockdown cities, total expenditure and utilisation frequency declined by $40.4 \%$ (95\% CI: $-58.1 \%$ to $-22.7 \%$ ) and $48.2 \%$ (95\% CI: $-49.9 \%$ to $-46.5 \%)$, while per capita expenditure increased by $4.3 \%$ (95\% CI: $-9.1 \%$ to $17.8 \%$ ) during the post-festival period in 2020. The trends in healthcare service utilisation in Eastern, Central and Western China are shown in table 1 and figure 1. Compared with 2019, total healthcare expenditure and utilisation frequency increased before the festival and decreased during and after the festival in 2020 in Eastern, Central and Western China. The greatest decrease was seen in Western China where total healthcare expenditure and utilisation frequency decreased by $40.8 \%$ (95\% CI: $-43.1 \%$ to $-38.4 \%$ ) and $43.3 \%$ (95\% CI: $-45.1 \%$ to $-41.4 \%$ ), respectively, during the post-festival period in 2020. Compared to 2019, per capita expenditure decreased in Eastern and Central China, but increased during the pre-and postfestival periods in 2020 in Western China.

\section{DID analysis}

Table 2 summarises our regression results. We estimated the impact of the COVID-19 pandemic on total healthcare expenditure, utilisation frequency and per capita expenditure during the pre-festival, festival and postfestival periods of 2020 in different treatment groups (medium-risk and high-risk cities and cities located in Hubei province) relative to the control cities (low-risk cities) in Panels A, B and C, respectively. The definition of the variable Treat is described in the section of statistical analysis. 

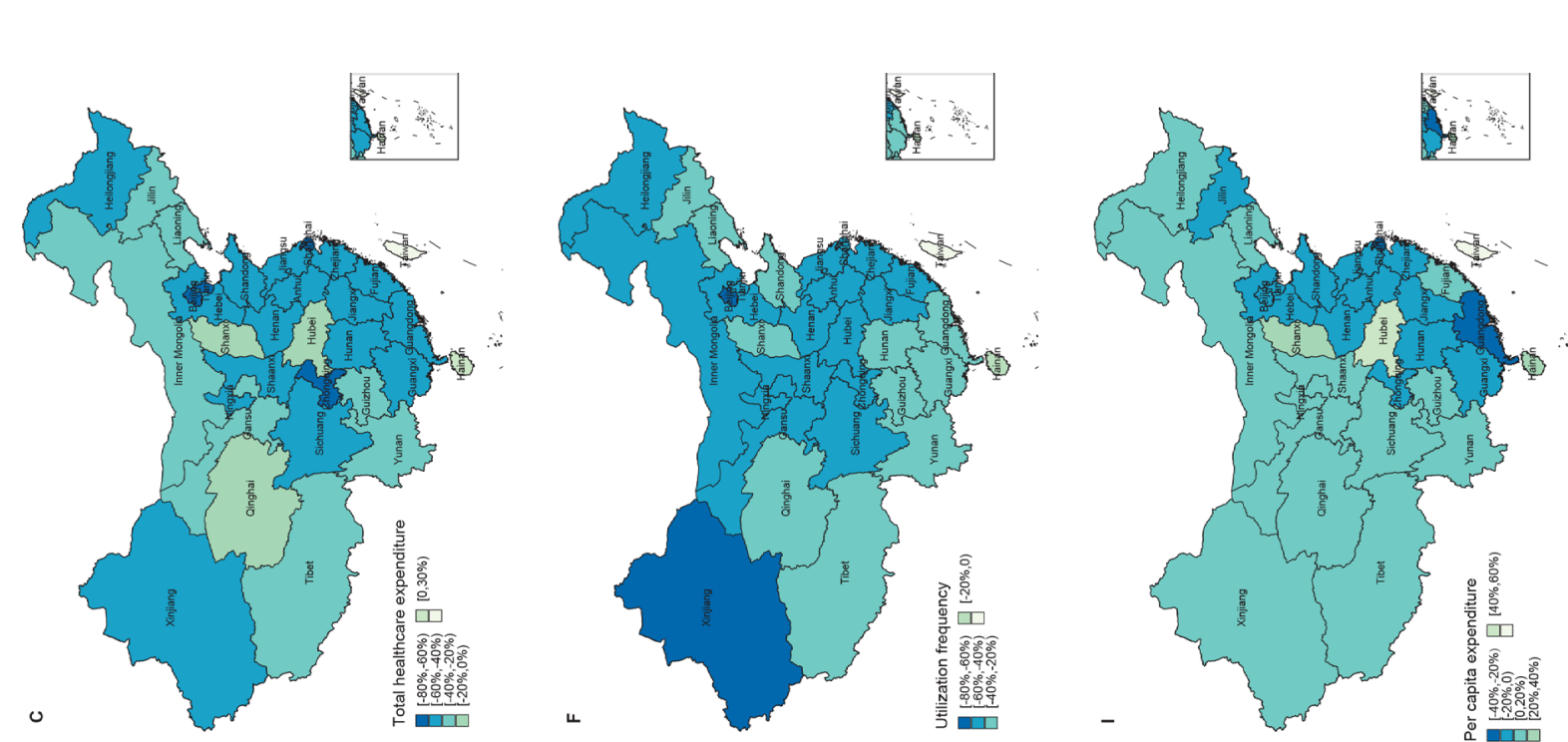

1)

de

응 응

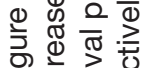

政造

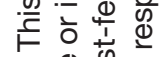

is

인 응

क्षे $\frac{1}{\pi} \frac{\pi}{0}$

ช

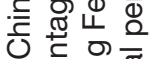

प

这的退

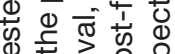

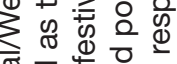

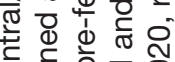
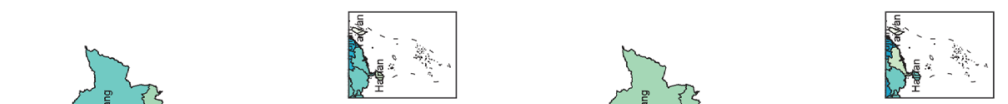

a.

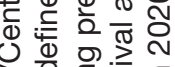

음

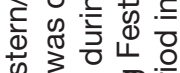

芩

的专

क

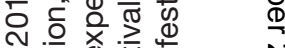

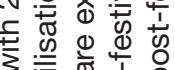

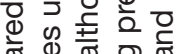

ब.

हो

O०

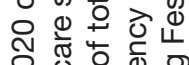

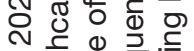

范

은 든

要

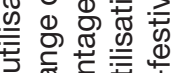
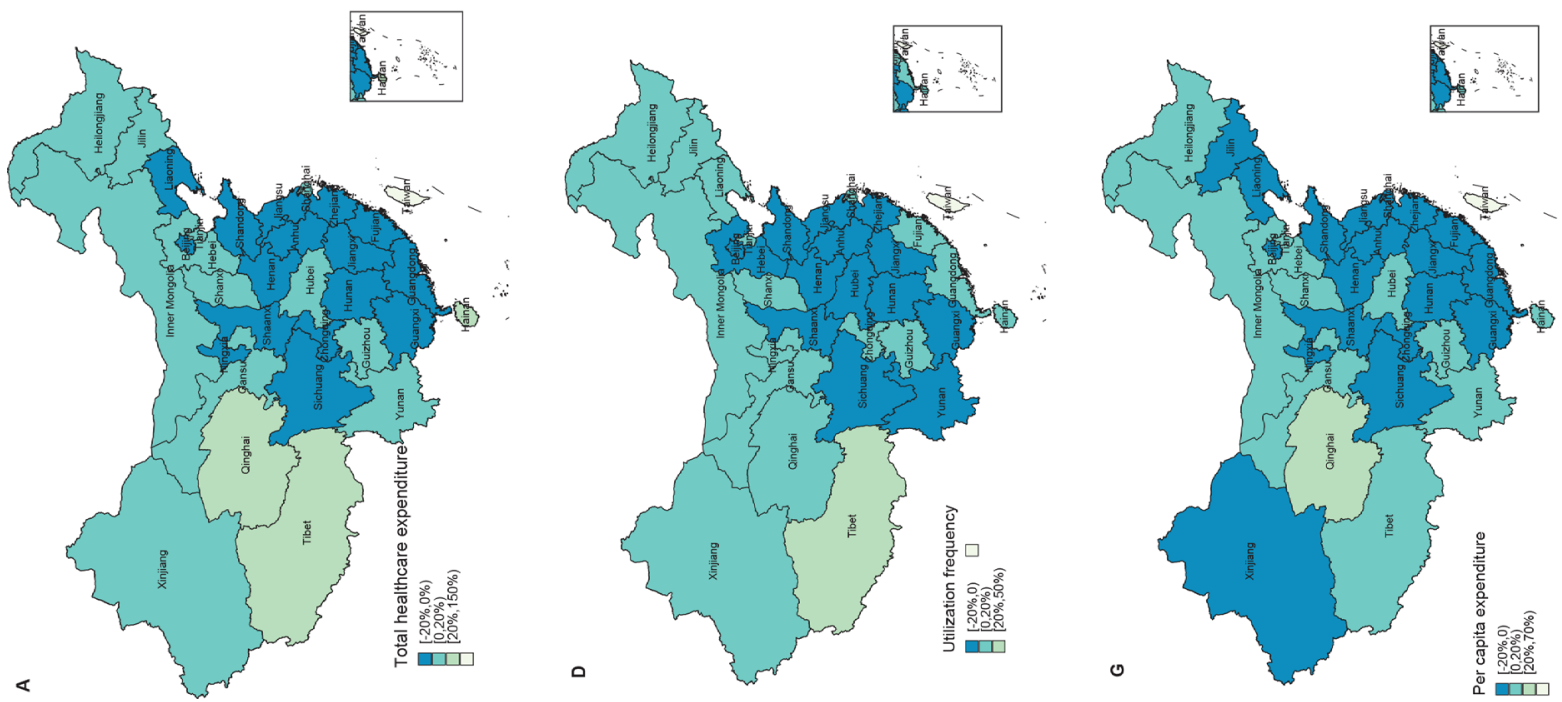

ब क $\frac{\pi}{0}$ के

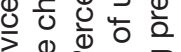

\%

要茯

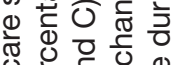

ชू

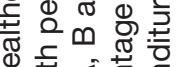

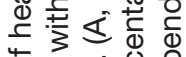

年

ब.

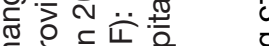

额

舫

ช

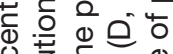

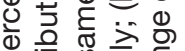

远

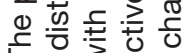

$\vdash .030$

- क्वे

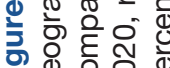


Table 2 Effects of COVID-19 on utilisation of healthcare services (\%)

\begin{tabular}{|c|c|c|c|}
\hline & $\begin{array}{l}\text { Total healthcare } \\
\text { expenditure }\end{array}$ & Utilisation frequency & $\begin{array}{l}\text { Per capita } \\
\text { expenditure }\end{array}$ \\
\hline \multicolumn{4}{|c|}{ Panel A (medium-risk vs low-risk cities) } \\
\hline Treat $_{\mathrm{i}}{ }^{*}$ Week $_{\mathrm{t}}{ }^{1}$ & $-4.4^{*}(2.4)$ & $-3.7^{\star * \star}(1.1)$ & $0.1(2.1)$ \\
\hline Treat $_{\mathrm{i}}{ }^{*}$ Week $_{\mathrm{t}}^{2}$ & $-1.4(2.9)$ & $-4.6^{\star \star \star}(1.6)$ & $6.3(4.0)$ \\
\hline Treat $_{\mathrm{i}}{ }^{*}$ Week$_{\mathrm{t}}^{3}$ & $-14.8^{\star \star \star}(2.6)$ & $-8.6^{\star \star \star}(0.9)$ & $-7.3^{\star \star}(3.0)$ \\
\hline $\mathrm{R}^{2}$ & 0.415 & 0.750 & 0.177 \\
\hline Observations & 5688 & 5688 & 5688 \\
\hline No of cities & 316 & 316 & 316 \\
\hline \multicolumn{4}{|c|}{ Panel B (high-risk vs low-risk cities) } \\
\hline Treat $_{\mathrm{i}}^{*}$ Week $_{\mathrm{t}}^{1}$ & $-5.1^{*}(2.7)$ & $-4.0^{\star \star \star}(1.3)$ & $-1.0(2.5)$ \\
\hline Treat $_{\mathrm{i}}^{*}$ Week $_{\mathrm{t}}^{2}$ & $-10.4^{\star \star \star}(3.1)$ & $-11.1^{\star \star \star}(2.1)$ & $-5.5(4.5)$ \\
\hline Treat $_{i}^{*}$ Week $_{t}^{3}$ & $-26.4^{\star \star \star}(2.7)$ & $-15.9^{* * *}(1.1)$ & $-18.4^{\star \star \star}(3.4)$ \\
\hline $\mathrm{R}^{2}$ & 0.344 & 0.690 & 0.154 \\
\hline Observations & 3114 & 3114 & 3114 \\
\hline No of cities & 173 & 173 & 173 \\
\hline \multicolumn{4}{|c|}{ Panel C (Hubei vs low-risk cities) } \\
\hline Treat $_{\mathrm{i}}^{*}$ Week $_{\mathrm{t}}^{1}$ & $21.2(17.8)$ & $-6.6^{\star \star \star}(2.0)$ & $29.2(18.3)$ \\
\hline Treat $_{\mathrm{i}}^{*}$ Week $_{\mathrm{t}}^{2}$ & $-11.1(11.2)$ & $-13.6^{\star \star \star}(3.0)$ & $12.2(13.1)$ \\
\hline Treat $_{i}^{*}$ Week $_{t}^{3}$ & $-27.5^{\star \star \star}(7.7)$ & $-24.4^{\star \star \star}(2.0)$ & $10.6(8.3)$ \\
\hline $\mathrm{R}^{2}$ & 0.311 & 0.668 & 0.168 \\
\hline Observations & 2880 & 2880 & 2880 \\
\hline No of cities & 160 & 160 & 160 \\
\hline Fixed effects & \multicolumn{3}{|c|}{ City fixed effects and time fixed effects in panel $A$, panel $B$ and panel $C$} \\
\hline
\end{tabular}

This table reports the results of estimating equation (1). The control and treatment groups for panels $A, B$ and $C$ are described in the text. City fixed effects and time fixed effects are included in panels A, B and C. SEs of coefficients are clustered at week level and reported in the parentheses behind the coefficients.

*Significant at the $10 \%$ level; ** significant at the $5 \%$ level; *** significant at the $1 \%$ level.

In Panels A, B and C, we found that the COVID-19 outbreak resulted in a decrease in utilisation of healthcare services as follows: (1) In medium-risk cities, the pandemic induced a $14.8 \%$ reduction in total healthcare expenditure and a $7.3 \%$ reduction in per capita expenditure during the post-festival in 2020 when including a set of fixed effects, and a $3.7 \%, 4.6 \%$ and $8.6 \%$ reduction in utilisation frequency before, during and after the festival in 2020, respectively. (2) In high-risk cities, the pandemic induced a $10.4 \%$ and $26.4 \%$ reduction in total healthcare expenditure during and after the festival in 2020 when including a set of fixed effects, respectively; and a $4.0 \%, 11.1 \%$ and $15.9 \%$ reduction in utilisation frequency before, during and after the festival in 2020, respectively; and $18.4 \%$ reduction in per capita expenditure during the post-festival period in 2020. (3) In cities located in Hubei, the pandemic induced a $27.5 \%$ reduction in total healthcare expenditure and a $24.4 \%$ reduction in utilisation frequency after the festival in 2020, respectively, when including a set of fixed effects. The results show that the COVID-19 outbreak decreased utilisation of healthcare services and that the higher the risk of COVID-19 in the city, the greater the impact.
We tested the parallel trend assumption for DID estimators and plotted the dynamics of the relationship between COVID-19 and the utilisation of healthcare services (figure 2). In Panels A, B and C, we found that there were no significant differences in total healthcare expenditure, utilisation frequency and per capita expenditure trends between the treatment group and control group for week $t$ in 2019. In medium-risk cities (figure 2A-C), utilisation of healthcare services declined immediately during the post-festival period (Week $t \geq 5$ ) in 2020 after the COVID-19 outbreak, such that $\beta_{t}$ is negative and significant at the $5 \%$ level. The greatest impact of the COVID-19 outbreak on total healthcare expenditure, utilisation frequency and per capita expenditure was $-20.1 \%,-9.2 \%$ and $-16.0 \%$, respectively. In highrisk cities (figure 2D-F), utilisation of healthcare services declined immediately during the festival and post-festival periods (Week $t \geq 4$ ) in 2020 after the COVID-19 outbreak, such that $\beta_{t}$ is negative and significant at the $5 \%$ level. The greatest impact of the COVID-19 outbreak on total healthcare expenditure, utilisation frequency and per capita expenditure were $-34.5 \%,-18.2 \%$ and $-30.2 \%$, respectively. In cities located in Hubei province (figure 2G-I), 

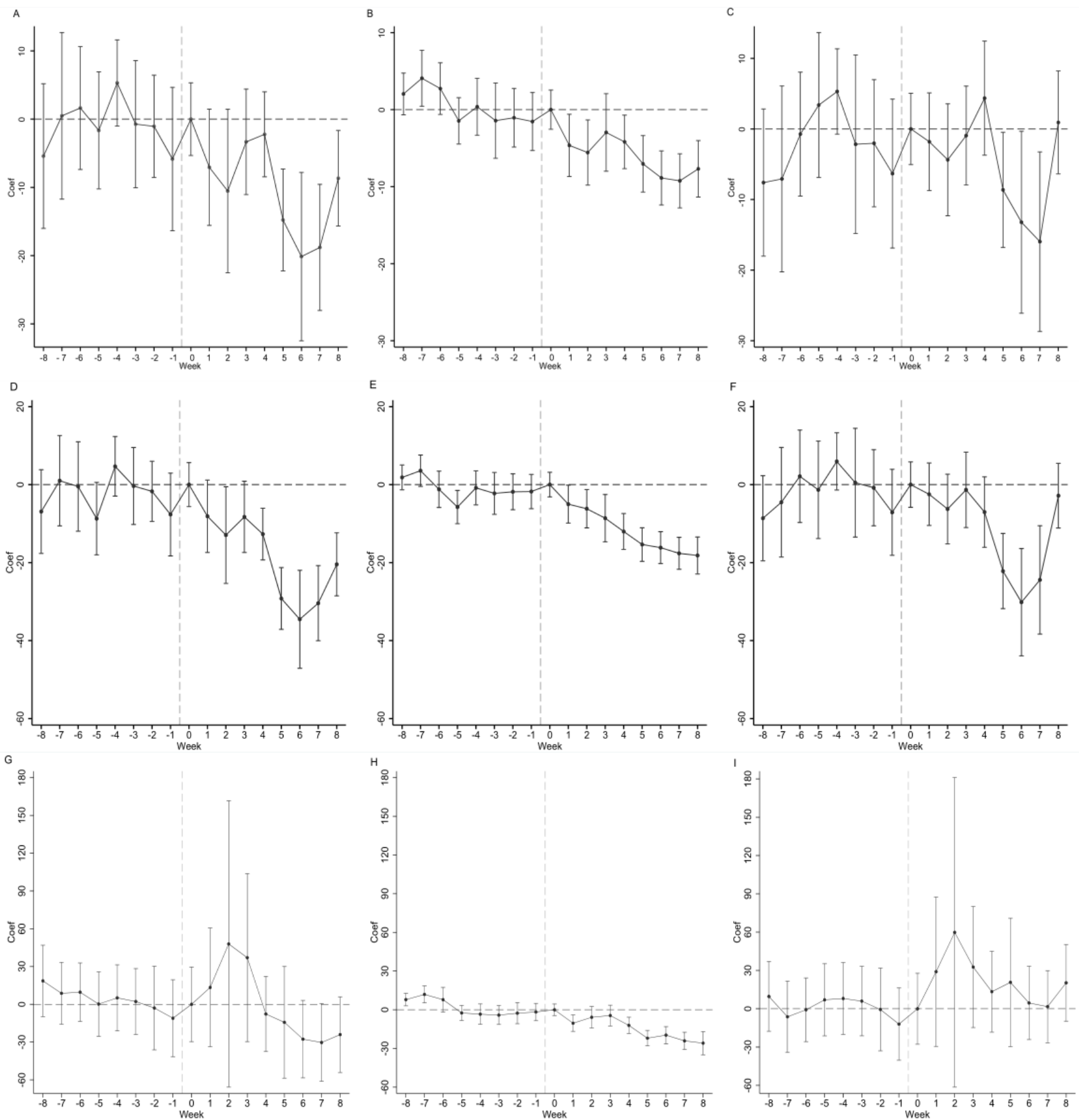

Figure 2 The dynamic impact of the COVID-19 on healthcare service utilisation in panels A/B/C. Notes: The figures plot the impact of COVID-19 on healthcare service utilisation in panels A/B/C. We included 2019 and 2020 dummies in the regressions, the left side of dotted line is 2019 and the right side is 2020 . The estimated coefficients and their $95 \% \mathrm{Cls}$ were plotted. A, B and C were the parallel trend tests of average weekly total healthcare expenditure, utilisation frequency and per capita expenditure in panel $A$, respectively. D, E and $F$ were the parallel trend tests of average weekly total healthcare expenditure, utilisation frequency and per capita expenditure in panel $\mathrm{B}$, respectively. $\mathrm{G}, \mathrm{H}$ and I were the parallel trend tests of average weekly total healthcare expenditure, utilisation frequency and per capita expenditure in panel $\mathrm{C}$, respectively. Specifically, we report estimated coefficients from the following regression: $Y_{i, t}=\beta_{0}+\beta_{1}$ Treat $_{i}^{*}$ Week $_{t}{ }^{1}+\beta_{2}$ Treat $_{i}^{*}$ Week $_{t}{ }^{2}+\ldots$ $+\beta_{17}$ Treat $_{i}^{*}$ Week $_{t}{ }^{17}+d_{i}+d_{t}+\varepsilon_{i, t}$ where $i(i=1, \ldots, 363)$ denotes the city, $t(t=-9, \ldots,-1,0,1, \ldots, 8)$ denotes the week, the added variables Week $_{t}^{J}(j=1, \ldots, 17)$ are the dummy variables of week $j$ that equals 1 when week $t=j$ and equals 0 otherwise, and the omitted benchmark week is the first week in 2019 (ie, $t=-9$ ). Treat is the treatment variable of city $i$ that equals 1 when $i$ is medium-risk, high-risk city and city in Hubei and equals 0 otherwise, $Y_{i, t}$ is the healthcare service utilisation in city $i$ during week $t$. The city-specific fixed effects $d_{i}$ control for unobserved city-specific heterogeneities. The week-specific fixed effects $d_{t}$ control for unobserved time-specific factors, including travel related to the Spring Festival (a time with high rates of travel in China). 

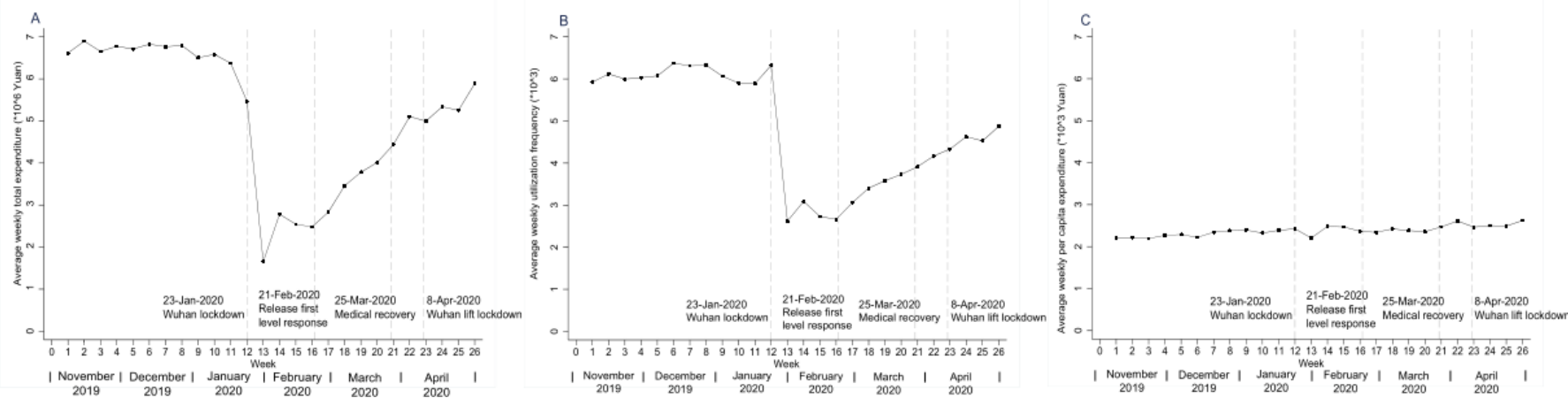

Figure 3 Trends for the utilisation of healthcare services during November 2019 to April 2020 in 365 mainland cities in China. Notes: The Spring Festival week is 25 January 2020 to 1 February 2020. Weeks 1-12 is pre-outbreak period (1 November 2019 to 22 January 2020), weeks 13-16 is during outbreak period (23 January 2020 to 20 February 2020), and weeks 17-26 is postoutbreak period (21 February 2020 to 30 April, 2020). (A) Trend for the total healthcare expenditure during November 2019 to April 2020 in China; (B) Trend for utilisation frequency during November 2019 to April 2020 in China; (C) Trend for per capita expenditure during November 2019 to April 2020 in China.

only utilisation frequency declined immediately during the festival and post-festival periods (Week $t \geq 4$ ) in 2020 after the COVID-19 outbreak, such that $\beta_{t}$ is negative and significant at the $5 \%$ level. The greatest impact of the COVID-19 outbreak on utilisation frequency was $-25.9 \%$.

\section{Trends in healthcare service utilisation between November 2019 and April 2020 in China}

We tracked healthcare service utilisation between November 2019 and April 2020 in 365 cities in China (figure 3). Total expenditure and utilisation frequency sharply declined in week 13 during the Spring Festival period and after the COVID-19 outbreak, compared with the normal levels seen during the pre-outbreak period (1 November 2019 to 22 January 2020, week 1 to week 12), and remained low throughout the COVID-19 outbreak (week 13-16). Once the pandemic was under control in China, some provinces lifted restrictions and lockdown measures, which resulted in an increase in total expenditure and utilisation frequency after 21 February 2020 (weeks 17-26). By the last week of April (week 26), total expenditure and utilisation frequency resumed to $89.3 \%$ and $79.9 \%$ of previous levels. Compared with normal levels, per capita expenditure declined during the festival (week 13) and increased after the festival (weeks 14-26). This increase could be explained by the fact that individuals who had delayed treatment due to COVID-19 sought care as soon as they could once the outbreak was over and restrictions were lifted.

\section{DISCUSSION}

This study assesses the difference in healthcare service utilisation before, during and after the Spring Festival in 2019 and 2020 to measure the impact of COVID-19 in China. Total healthcare expenditure and utilisation frequency declined sharply during the post-festival period in 2020 when the pandemic was at its peak and many cities were under strict lockdown. A greater decrease was found in high-risk cities, lockdown cities and in cities located in Western China. The DID results imply that, compared with the low-risk cities, the pandemic induced a significant decrease in the healthcare utilisation during the Spring Festival and post-festival period in 2020 relative to 2019, especially in high-risk cities.

Our findings suggest that healthcare service utilisation decreased during and after the COVID-19 outbreak. This decrease could be explained by the fact that resources (including human resources) were reallocated to battle COVID-19, leaving less capacity to address other health services during the outbreak. Similar patterns have emerged in other COVID-19 torn countries such as the UK, the USA, France, Spain and Italy. ${ }^{22}{ }^{23}$ Life-saving treatments such as organ transplants have decreased dramatically, and there have been reports of sharp decreases in emergency room visits. ${ }^{24}$ Hong Kong found patients waited for around 5 hours before seeking care in early 2020 compared with only waiting around 1 hour during the same period in $2019 .{ }^{23}$ Patients may delay seeking health services for multiple reasons including the fear of nosocomial infection, limitations imposed by lockdown restrictions and/or due the belief that services should be reserved for patients with COVID-19. Prevention services are also suffering as routine immunisation campaigns are suspended in light of COVID-19. ${ }^{25}$ Given this gap in services, we could continue to see long-term negative consequences on health conditions due to delayed treatment or prevention.

Our findings also suggest that this interruption in access to healthcare services will outlast the COVID-19 outbreak. By the last week of April 2020, even as the pandemic was under control, utilisation had only gradually increased to $80 \%-89 \%$ of the normal levels seen during the pre-outbreak period during 1 November 2019 to 22 January 2020. These patterns are not unprecedented. Other major public health events have caused similar disruptions to healthcare services. For example, as already mentioned Ebola caused great upheaval in West Africa, and even areas with few cases of Ebola and where health facilities remained open there was a $30 \%$ decline in facility-based healthcare. ${ }^{11} 1326$ 
These negative indirect effects could further exacerbate disparities in access to healthcare in different regions in China. We found that the pandemic had a greater negative impact in China's Western cities which are less developed with less resources than Eastern or Central China. ${ }^{27}$ For example, according to the 2018 China Health Statistics Yearbook, there are only 23 and 21 physicians per 10000 residents in the Guizhou and Yunnan province (part of the Western region in China) respectively, while Beijing and Shanghai in Eastern China had 46 and 30 physicians per 10000 residents, respectively. Healthcare workers from all around the country were sent to Hubei province to help tackle the pandemic, leaving many hospitals with fewer staff and resources. ${ }^{7}$ In addition, since most healthcare services and resources in the Western region are concentrated in a few cities, travel restrictions, and supply chain interruptions caused by the pandemic further complicated access to healthcare services in the more rural areas.

Finally, the decrease in healthcare service utilisation may have a lasting financial impact on the health system. Payment for medicine and services is a major source of revenue for public hospitals in China. The significant decrease in revenue may affect the operation of all health service providers, especially in the Western region where the healthcare system is more financially vulnerable. ${ }^{28}$ Financial hardships will only serve to further negatively impact the health of affected populations and exasperate inequalities.

This study has some limitations. First, we were unable to assess the overall utilisation of healthcare services because we could not directly compare CUP card usage between 2019 and 2020, and CUP cards were not the only method of payment for health services. Therefore, the reduction in total healthcare expenditure and utilisation frequency may be underestimated. After the outbreak, the usages of other transaction mechanisms (such as WeChat and Ali Pay) might increase, but the increment of proportion should be relatively small during the short time of our study period in healthcare settings. To try and weaken confounding effects from other transaction mechanisms, we used the relative value of total healthcare expenditure, utilisation frequency or per capita expenditure as the outcomes. We assumed that the change in the proportion of new finance transaction mechanisms and cash payments in healthcare services were negligible during the relatively short time of our study period. Under that assumption, their possible confounding factors were eliminated in the DID model further. Second, we did not examine which specific types of healthcare services were underused. Understanding which health issues were most or least affected could help with resource allocation during future public health emergencies. Third, all cities in China imposed strict control measures which limited movement and access to health services regardless of the number of COVID-19 cases in the cities, and a majority of healthcare services were suspended during the outbreak as resources had to be reallocated to address COVID-19.
Therefore, we were not able to make comparisons to a real control group unaffected by the pandemic. This might lead to an underestimation of the effect. In addition, we had to exclude two cities due to lack of data in the DID analysis which may reduce the representativeness of this study. However, the two cities were small with relatively low health service expenditure, and therefore their exclusion should have little effect on our findings.

To our knowledge, this is the first study to quantify and track the impact of COVID-19 on the utilisation of healthcare services in all 365 mainland cities of China. The pandemic significantly reduced healthcare service utilisation regardless of the level of COVID-19 risk in cities, emphasising the national and global impact of pandemics on healthcare. While utilisation has increased during the post-outbreak period, this increase has been slow.

\section{CONCLUSION}

Efforts to maintain essential and primary healthcare should be strengthened during public health emergencies. Our findings suggest that countries need to identify methods to minimise disruption in treatment and prevention of non-pandemic-related health concerns as much as possible and have plans on how to resume prepandemic levels as quickly as possible. These policies take into consideration patient reluctance to seek healthcare during a pandemic and effort should be made to ensure patients feel protected from nosocomial transmission. Furthermore, public messaging should not to dissuade patients from seeking care, especially for essential treatment. Furthermore, strategies to prevent the spread of a pandemic such as lockdown restrictions need to make allowances for healthcare service utilisation. All these issues will require policy-makers to conduct careful risk-benefit analyses for developing and implementing mitigations strategies. Future research is required to fully measure the impact emanating from the disruption in healthcare services on the health of individuals, especially the poor and the vulnerable in years to come.

Contributors C-WF, X-HY and S-LT developed the research question. C-WF, X-HY and $\mathrm{Y}$-NZ oversaw study implementation. Y-NZ and $\mathrm{YC}$ were responsible for the data analysis and drafting the manuscript. C-WF, S-LT, YW, FL, MP, NW, FY, and X-HY assisted with the study design, data analysis, data interpretation and reviewed the manuscript. The authorship order among cofirst authors was assigned according to contributions. All authors read and approved the final manuscript.

Competing interests None declared.

Patient consent for publication Not required.

Provenance and peer review Not commissioned; externally peer reviewed.

Data availability statement Data are available on reasonable request from C-W Fu emailed to fcw@fudan.edu.cn. The data are daily transactions for healthcare services and include information on each transaction including date, amount, location and name of the healthcare facility in 365 mainland Chinese cities during 8 January to 12 March 2019 and 1 November 2019 to 30 April 2020. The data are available on reasonable request.

Supplemental material This content has been supplied by the author(s). It has not been vetted by BMJ Publishing Group Limited (BMJ) and may not have been peer-reviewed. Any opinions or recommendations discussed are solely those of the author(s) and are not endorsed by BMJ. BMJ disclaims all liability and 
responsibility arising from any reliance placed on the content. Where the content includes any translated material, BMJ does not warrant the accuracy and reliability of the translations (including but not limited to local regulations, clinical guidelines, terminology, drug names and drug dosages), and is not responsible for any error and/or omissions arising from translation and adaptation or otherwise.

Open access This is an open access article distributed in accordance with the Creative Commons Attribution Non Commercial (CC BY-NC 4.0) license, which permits others to distribute, remix, adapt, build upon this work non-commercially, and license their derivative works on different terms, provided the original work is properly cited, appropriate credit is given, any changes made indicated, and the use is non-commercial. See: http://creativecommons.org/licenses/by-nc/4.0/.

ORCID iD

Yun Chen http://orcid.org/0000-0002-2516-0946

\section{REFERENCES}

1 Zhu N, Zhang D, Wang W, et al. A novel coronavirus from patients with pneumonia in China, 2019. N Engl J Med 2020;382:727-33.

2 WHO. Coronavirus disease (COVID-19) pandemic, 2020. Available: https://www.who.int/emergencies/diseases/novel-coronavirus-2019 [Accessed 2 Sep 2020].

3 JHU. COVID-19 dashboard by the center for systems science and engineering (CSSE) at Johns Hopkins University (JHU). Available: https://coronavirus.jhu.edu/map.html [Accessed 23 May 2020].

4 Chen S, Yang J, Yang W, et al. COVID-19 control in China during mass population movements at new year. Lancet 2020;395:764-6.

5 Amos $\mathrm{O}$. The cancer patient trapped by coronavirus: what happened next? BBC News. Available: https://www.bbc.com/news/world-asia51748164 [Accessed 7 Mar 2020].

6 Deng C. People without coronavirus say Wuhan doctors too busy for them. WSJ. Available: https://www.wsj.com/articles/ patients-without-coronavirus-struggle-to-get-urgent-care-in-china11582134713 [Accessed 19 Feb 2020].

7 Bloomberg. Hospitals in China, Overwhelmed by coronavirus, turn away patients with other Pressing needs. time. Available: https:// time.com/5788495/china-hospital-shortage/ [Accessed $20 \mathrm{Feb}$ 2020].

8 Liebensteiner MC, Khosravi I, Hirschmann MT, et al. Massive cutback in orthopaedic healthcare services due to the COVID-19 pandemic. Knee Surg Sports Traumatol Arthrosc 2020;28:1705-11.

9 Ayas M, Ali Al Amadi AMH, Khaled D, et al. Impact of COVID-19 on the access to hearing health care services for children with cochlear implants: a survey of parents. F1000Res 2020;9:690.

10 Parpia AS, Ndeffo-Mbah ML, Wenzel NS, et al. Effects of response to 2014-2015 Ebola outbreak on deaths from malaria, HIV/AIDS, and tuberculosis, West Africa. Emerg Infect Dis 2016;22:433-41.

11 Barden-O'Fallon J, Barry MA, Brodish P, et al. Rapid assessment of Ebola-Related implications for reproductive, maternal, newborn and child health service delivery and utilization in guinea. PLoS Curr 2015;7. doi:10.1371/currents. outbreaks.0b0ba06009dd091bc39ddb3c6d7b0826. [Epub ahead of print: 04 Aug 2015].

12 Bolkan HA, Bash-Taqi DA, Samai M, et al. Ebola and indirect effects on health service function in Sierra Leone. PLoS Curr 2014;6. doi:10.1371/currents. outbreaks.0307d588df619f9c9447f8ead5b72b2d. [Epub ahead of print: 19 Dec 2014].

13 Ly J, Sathananthan V, Griffiths T, et al. Facility-based delivery during the Ebola virus disease epidemic in rural Liberia: analysis from a cross-sectional, population-based household survey. PLoS Med 2016;13:e1002096.

14 Takahashi S, Metcalf CJE, Ferrari MJ, et al. Reduced vaccination and the risk of measles and other childhood infections post-Ebola. Science 2015;347:1240-2.

15 Delamou A, Ayadi AME, Sidibe S, et al. Effect of Ebola virus disease on maternal and child health services in guinea: a retrospective observational cohort study. Lancet Glob Health 2017;5:e448-57.

16 Glied S, Levy $\mathrm{H}$. The potential effects of coronavirus on national health expenditures. JAMA 2020;323:2001.

17 Fang Z, Zhu J. How unionpay quick pass is beating alipay and WeChat pay in China. Cornwall: UKTJ International Ltd, 2020: 155-7.

18 He G, Pan Y, Tanaka T. COVID-19, City Lockdowns, and air pollution: evidence from China. medRxiv 2020

19 Angrist J, Pischke JS. Mostly harmless econometrics: an empiricist's companion. Princeton: Princeton University Press, 2009: 165-84.

20 Jacobson L, Lalonde RJ, Sullivan DG. Earnings losses of displaced workers. Am Econ Rev 1992;83:685-709.

21 Team RC. R: a language and environment for statistical computing R foundation for statistical computing, Vienna, Austria, 2020. Available: https://www.r-project.org/ [Accessed 20 Aug 2020].

22 Holmes JL, Brake S, Docherty M, et al. Emergency ambulance services for heart attack and stroke during UK's COVID-19 lockdown. Lancet 2020;395:e93-4.

23 Spinney L. Concern as heart attack and stroke patients delay seeking help. The guardian. Available: https://www.theguardian com/world/2020/apr/16/coronavirus-concern-heart-attack-strokepatients-delay-seeking-help [Accessed 16 Apr 2020].

24 Loupy A, Aubert O, Reese PP, et al. Organ procurement and transplantation during the COVID-19 pandemic. Lancet 2020;395:e95-6.

25 Initiative MR. More than 117 million children at risk of missing out on measles vaccines, as COVID-19 surges. Available: https://measlesr ubellainitiative.org/measles-news/more-than-117-million-childrenat-risk-of-missing-out-on-measles-vaccines-as-covid-19-surges/ [Accessed 23 May 2020].

26 Brolin Ribacke KJ, van Duinen AJ, Nordenstedt H, et al. The impact of the West Africa Ebola outbreak on obstetric health care in Sierra Leone. PLoS One 2016;11:e0150080.

27 Fang P, Dong S, Xiao J, et al. Regional inequality in health and its determinants: evidence from China. Health Policy 2010:94:14-25.

28 Yip W, Fu H, Chen AT, et al. 10 years of health-care reform in China: progress and gaps in universal health coverage. Lancet 2019;394:1192-204. 